\title{
PATRÓN DE CONDUCTA TIPO A Y B, Y SU RELACIÓN CON LAS ADICCIONES CONDUCTUALES
}

\author{
TYPE A \& B BEHAVIOR PATTERN AND ITS RELATION TO \\ BEHAVIORAL ADDICTIONS
}

\author{
Gisele Belmonte Steibel ${ }^{1}$, Rosario RuiZ-OLIVARES ${ }^{2}$ Y \\ JAVIER HERRUZO CABRERA ${ }^{2}$
}

Cómo referenciar este artículo/How to reference this article:

Belmonte Steibel, G., Ruiz-Olivares, R., y Herruzo-Cabrera, J. (2016). Patrón de Conducta Tipo A y $\mathrm{B}$, y su relación con las adicciones conductuales [Type A \& B Behavior Pattern and its relation to behavioral addictions]. Acción Psicológica, 13(1), 119-128 http://dx.doi.org/10.5944/ap.13.1.17430

\section{Resumen}

El objetivo de esta investigación fue relacionar el patrón de conducta Tipo A y B (PCTA y PCTB) con la posibilidad de presentar adicciones a Internet, juego, compras y abuso del teléfono móvil en una muestra de jóvenes de la provincia de Córdoba (España). Con un diseño prospectivo expost-facto con dos grupos, se elaboró un cuestionario con el que se recogieron datos sociodemográficos, y en el que se incluyeron otros cuestionarios como el Test de adicción a las compras, el Test de adicción a Internet de Echeburúa (2003), el Cuestionario Breve de Juego Patológico de Fernández-
Montalvo, Echeburúa y Baéz (1997); algunos ítems sobre el uso del teléfono móvil; y el cuestionario de PCTA de Jenkins. Participaron 3815 jóvenes de la provincia de Córdoba (España) con un rango de edad entre los 18 y 29 años. Los resultados indican que los jóvenes PCTA son los que más compran, juegan, se conectan a Internet y usan el móvil. En conclusión se puede inferir que los jóvenes con patrón de personalidad Tipo A tienen más posibilidades de presentar conductas adictivas que los jóvenes de patrón de conducta Tipo B.

Palabras clave: Patrón de Conducta Tipo A; Patrón de Conducta Tipo B; conductas adictivas sin sustancia; jóvenes.

Correspondencia: Javier Herruzo Cabrera. Departamento de Psicoloía. Facultad de Ciencias de la Educación, Universidad de Córdoba.

Email: jherruzo@uco.es

${ }^{1}$ Psicóloga do Centro de Atenção psicossocial de Álcool e outras drogas (CAPS-AD de Pelotas), Brasil.

${ }^{2}$ Departamento de Psicología (Universidad de Córdoba), España.

Recibido: 02 de enero de 2016.

Aceptado: 12 de febrero de 2016. 


\begin{abstract}
This research aimed to relate the possibility of submitting the Type A and the Type B behavior patterns to the no-substance addiction. We investigated the Internet addiction, the gambling, the shopping, and the misuse of mobile in a sample of youth people. With a prospective design ex-post-facto two groups, a questionnaire was developed to collect sociodemographic data, in which we included the Test of Shopping Addiction, the Test of Internet Addiction from Echeburúa (2003), the Pathological Gambling Brief Questionnaire from Fernandez-Montalvo Echeburúa y Baez (1997), some items on Mobile Phone Usage, and the Jenkins Activity Survey. Young people from the Province of Córdoba, Spain, have participated $(\mathrm{N}=3815$, age range between 18 and 29). Results indicated that young people characterized as Type A behavior pattern show bigger propension to shopping, to playing, to Internet connection, and to use of mobile phone. According to logistic regression, The Type B behavior pattern showed to be a protective factor for addiction to shopping. In conclusion it can be inferred that young people of Type A behavior pattern are more likely to have addictive behaviors that young people of Type B behavior pattern.
\end{abstract}

Keywords: Type A behavior pattern; Type B behavior pattern; no substance addictive behaviors; youth.

\section{Introducción}

Las tecnologías de la información y la comunicación (TICs) son de creciente interés en los medios académicos y de la salud, no sólo por su impacto en la sociedad sino por su uso abusivo. Los jóvenes suscitan especial interés por ser la población más vulnerable (Estevez, 2014; Sánchez-Carbonell, Beranuy, Castellana, Chamarro y Obrest, 2008; Ruiz-Olivares, Lucena, Pino y Herruzo 2010; Salehan y Negahban, 2013). La tecnología, como
Internet y el móvil, forman parte de un sistema de comunicación de una potencia incomparable con la tecnología anterior, pues permiten intercambiar informaciones y representaciones de forma instantánea, cambiando así la forma de relación con el mundo (García-Castillo, 2013; Viñas y González, 2010). En nuestra cultura el uso de la tecnología está desarrollándose rápido entre la población, no sólo se ha generalizado su uso, sino también el tiempo y los tipos de actividades que usan las TICs se han multiplicado (chat, compras, trabajo, etc.). La tecnología se ha convertido en uno de los reforzadores inmediatos más potente (Garcia-Castillo, 2013). Pero también este incremento ha hecho que cada vez con más frecuencia lleguen a consulta casos con un uso excesivo y problemático del uso de estas TIC's (Salehan y Negahban, 2013). De la misma manera que otras actividades cotidianas como el juego, el trabajo, las compras, el ejercicio físico, sexo, etc. son susceptibles de ser adictivas, el uso de las TIC's también puede llegar a ser problemático, interfiriendo y condicionando seriamente la vida de una persona (Coll y Monereo, 2008; Echeburúa y Corral, 2010; Echeburúa, Labrador y Becoña, 2009). Según algunos autores, el uso abusivo de las TICs puede producir sintomatología similar a la de otras adicciones, como disforia, dependencia y tolerancia. (Echeburúa y Corral, 2010; Gutiérrez, Valladolid y Fonseca, 2013; Labrador y Villadangos, 2010; Oliva et al., 2014).

Concretamente, en la literatura empírica el uso excesivo del teléfono móvil se define como la incapacidad para controlar o interrumpir su uso (Echeburúa et al., 2009; Muñoz-Rivas y Agustín, 2005; Sánchez-Carbonell et al., 2008). En algunos trabajos de investigación se ha encontrado que las mujeres jóvenes son las que mandan más mensajes, que no consiguen reducir los gastos y que pasan más horas hablando por él móvil (Carbonell et al., 2012; Hong, Chiu y Huang, 2012; Ruiz-Olivares et al., 2010). Según Perez, Monje, De León y Ruiz (2012), una gran parte de la literatura apunta algunas características psicológicas como precipitantes de dicho uso abusivo, como trastornos concomitantes, autoestima baja y experimentación de síntomas depresivos. Además del riesgo en sí mismo que presenta el uso excesivo del teléfono móvil, hay que añadir la facilidad con la que se accede a otro tipo de actividades también susceptibles de adicción, 
como las compras, el sexo, el juego, etc. (Echeburúa et al., 2009; Perez et al., 2012).

Comprar es una conducta normativa y adaptativa en condiciones normales, pero también es susceptible de generar adicción si se sobrepasan determinados límites (Echeburúa, 2003; García-Castillo, 2013). Personas que presentan una adicción a las compras son incapaces de controlar su vida, suelen consumir cosas de manera no planificada, excediéndose en sus posibilidades económicas. Estos perfiles mayormente se manifiestan en mujeres de 30 años, pero es en la juventud y adolescencia donde se inicia el problema (Becoña, 2003; Denegri, Sepúlveda y Godoy, 2011; Ruiz-Olivares et al., 2010).

Actualmente, el uso problemático de Internet se relaciona con el número de horas dedicado por ejemplo a las redes sociales, chat, páginas de contenido para adultos y películas (Carbonel et al., 2012; Oliva et al., 2014; Puerta-Cortés y Carbonell, 2014). Algunos trabajos de investigación señalan que jóvenes universitarios con un uso problemático de Internet presentaban una puntuación alta en rasgos de la personalidad como el neuroticismo, y baja puntuación en rasgos como la afabilidad y la responsabilidad (Kuss, Van Rooij, Shorter, Griffiths y Van de Mheen, 2013; Oliva et al., 2014; Puerta-Cortés y Carbonell, 2014). Sin embargo, la dificultad de delimitar conceptualmente este comportamiento anormal ha hecho que la más reciente revisión del Manual Diagnóstico y Estadístico de Trastornos Mentales (DSM-V) de la American Psychiatric Association (APA, 2013) no incluya como patología la adicción a Internet.

En cuanto a la adicción al juego, se puede decir que el perfil del jugador ha variado en estos últimos años, pasando de ser un hombre de 40 años de edad, a un de joven de entre 15 a 30 años, muchos de ellos estudiantes, que juegan cada vez más horas (Estévez, Herrero y Sarabia, 2013). El juego patológico es considerado una conducta adictiva que está clasificado en el grupo de los trastornos no relacionados con consumo de sustancias (APA, 2013). El jugador patológico no tiene capacidad para controlarse, el juego es el centro de su vida. Como argumentan Gutiérrez et al. (2013), el juego también activa sistemas de recompensa similares a los activados por el consumo de drogas, produciendo síntomas de com- portamientos similares a los producidos por los trastornos por uso de sustancias.

Algunas de las razones que se dan en la literatura para explicar el aprendizaje de este tipo de conductas potencialmente adictivas han sido las características individuales y de la personalidad de cada individuo (Becoña, 2006; Fernádez, Horrupia-Serrano y Blannxart, 2012; Puerta-Cortéz y Carbonell, 2014). No se puede afirmar que haya una causa única ni un trastorno de personalidad típico para las personas adictas, pero sí de ciertas características de la personalidad presentes en estos comportamientos (Fantín, 2006; Jimenéz, 2007; Salazar, Fraile y Bobes, 2010). Estas características pueden predisponer, precipitar o perpetuar el abuso y la dependencia de una sustancia, y también de conductas susceptibles de ser adictivas como comprar, jugar, usar el teléfono móvil, y/o Internet. Los rasgos de personalidad podrían considerarse factores preexistentes que rigen la elección de realizar una determinada conducta y perpetuar la realización de las mismas (Le Bona et al., 2004). Así, una característica de la personalidad clásicamente asociada a las adicciones ha sido la impulsividad y la búsqueda de placer rápido y fuga del estrés (Adan, 2012; Arteaga, FernándezMontalvo y López-Goñi, 2012; Becoña y Tomás, 2010; Doran, McChargue y Cohen, 2007; Gutiérrez et al., 2013). Uno de los patrones conductuales que más relación presenta con el estrés y con el ámbito de la salud es el patrón de conducta Tipo A (PCTA). Estas personas se caracterizan por tener una postura de alerta, una expresión facial tensa, el caminar rápido y el hacer las cosas apresuradamente, además de ciertas conductas interpersonales como interrumpir a los demás y retarlos (DiazCosta, 2007; Lahan, 2008; Palermo y León, 1993; Rodriguez-Sutil, Gil-Corbacho y Martiñez, 1996). Como versus al PCTA, se encuentra el PCTB que se caracteriza por ser una persona con una menor reactividad fisiológica frente a situaciones de estrés y en una no hiperactividad motora (Gutiérrez, 2001; Jenkins, 1992; Pulido y Fonseca, 2009). Algunos trabajos de investigación han relacionado el PCTA y PCTB con una posible adicción a los videojuegos. En este caso, parece que los participantes Tipo A tenían el doble de probabilidades que los participantes Tipo B para cumplir con los criterios de adicción a los videojuegos (Griffiths y Dancaster, 1995). 
A pesar de la escasa literatura científica que relacione el PCTA con determinadas conductas adictivas como navegar por Internet, comprar, jugar y usar el móvil se hace necesaria la realización de investigaciones que profundicen en esta relación. Tal conocimiento podría facilitar la promoción de la salud y la disminución de futuros problemas adictivos en la población joven, además de planificar tratamientos más eficaces y adecuados.

Así, el objetivo de este trabajo de investigación fue relacionar el patrón de conducta Tipo A (PCTA) y Tipo $\mathrm{B}$ (PCTB) con posibles conductas adictivas como Internet, uso del móvil, compras y juego en jóvenes de la provincia de Córdoba (España).

\section{Método}

\section{Participantes}

Participaron en este estudio 3815 jóvenes de la ciudad y provincia de Córdoba, donde 1410 (40.12\%) eran de la ciudad y $2105(59.87 \%)$ de zonas rurales. De los 3815 jóvenes que respondieron el cuestionario, sólo fueron analizados $801(21.00 \%)$ participantes que habían completado correctamente el cuestionario de Jenkins y cumplían los requisitos para PCTA o PCTB. Así, 247 sujetos eran PCTA (30.80\%), de los cuales 189 eran de zonas rurales $(76.50 \%)$ y 58 de zona urbana $(23.50 \%)$. Por otro lado 554 sujetos eran PCTB $(69.20 \%)$, de los cuales 412 eran de zonas rurales $(74.40 \%)$ y 142 de zona urbana $(25.60 \%)$. En cuanto a la variable 'sexo', 139 hombres $(57.00 \%)$ y 105 mujeres $(43.00 \%)$ eran PCTA, y 209 hombres $(37.80 \%)$ y 344 mujeres $(62.20 \%)$ eran PCTB. En función de la variable 'edad', y teniendo en cuenta los participantes PCTA, la muestra se distribuía en 75 participantes de 18-20 años (30.60\%), 69 de 21 a 23 años $(28.20 \%), 50$ de 24 a 26 años (20.40\%), 43 de 27 a 29 años (17.60\%) y 8 mayores de 29 años $(3.30 \%)$. En función de los participantes PCTB, la muestra se distribuía en 221 participantes de $18-20$ años (40.30\%), 128 de 21 a 23 años (23.30\%), 98 de 24 a 26 años (17.90\%), 77 de 27 a 29 años (14.00\%) y 25 mayores de 29 años (4.60\%).

\section{Instrumentos de evaluación}

El cuestionario utilizado ha sido una adaptación del que se ha aplicado en otros trabajos de investigación con población universitaria de la provincia de Córdoba (RuizOlivares et al., 2010; Ruiz-Olivares, Lucena, Pino, Raya y Herruzo, 2010). En él se incluyeron datos sociosdemográficos y otros instrumentos para valorar el uso de las compras, juego, uso de Internet y móvil. Para valorar el abuso de las compras, se utilizó el Test de Adicción a las Compras de Echeburúa (2003), formado por cinco ítems, con dos alternativas de respuesta "si" o "no", considerando que la persona tiene un problema de adicción a las compras si responde afirmativamente a dos o más ítems. Para valorar el comportamiento relacionado con el juego patológico, se utilizó el Cuestionario Breve de Juego Patológico (CBJP) de Fernández-Montalvo, Echeburúa y Báez (1995), formado por 4 ítems, con dos alternativas de respuesta "si" o "no", considerando que la persona tiene un problema con el juego si responde afirmativamente a dos o más ítems. En el trabajo de RuizOlivares et al. (2010) fue calculado el índice de fiabilidad del cuestionario, $\alpha$ de Cronbach, obteniendo un valor de 0.94 , con lo que se puede decir que la fiabilidad del instrumento es adecuada. Para valorar la adicción al uso de Internet, se ha utilizado el Test de Adicción a Internet (Echeburúa, 2003), formado por 9 ítems, con dos alternativas de respuesta "si" o "no", con una escala de puntuación dependiendo del número de respuestas afirmativas (Echeburúa, 2003, p. 70). Así, de 0-3 respuestas afirmativas se considera que la persona no tiene problemas con el uso de Internet, de 4-6 respuestas afirmativas se considera que la persona está en riesgo de tener una dependencia y de 7-9 respuestas afirmativas es probable que la persona presente un problema de adicción a Internet. Para valorar el uso del teléfono móvil, se incluyeron una serie de ítems que hacían referencia al tiempo en horas que pasaban hablando o al número de mensajes que mandaban al día los estudiantes (Bianchi y Phillips, 2005). En el trabajo de Ruiz-Olivares et al. (2010), se calculó también el índice de fiabilidad $\alpha$ de Cronbach para este instrumento obteniendo un índice de 0.93 , lo que indica que la validez del instrumento es adecuada.. Así, el cuestionario quedó dividido en un bloque de datos sociodemográficos y otro donde se 
obtenía información acerca de patrones de conducta relacionados con el uso de Internet, las compras, el teléfono móvil y el juego.

Por otro lado, para valorar el PCTA/PCTB se utilizó el Inventario de Actividad de Jenkins Forma H (JASE-H; Krantz, Glass y Zinder, 1974), validada la versión española frente al 'Inventario de Actividad de Jenkins Forma T' (Krantz et al., 1974), con un coeficiente $\alpha$ de Cronbach entre 0.75 y 0.88 . El JASE-H presenta 32 ítems en la forma de frases, a las cuales el sujeto debe señalar el grado de aplicabilidad y descripción que sobre sí mismo que aporta cada una. Las opciones de respuesta están formadas por una escala tipo Likert de 6 puntos, cuyo límite inferior (1 - "No en absoluto") y superior (6 "Total acuerdo") deben aplicarse a la situación reflejada en cada uno de los ítems. El JASE-H está diseñado para medir el PCTA según cuatro factores: la competitividad, la sobrecarga laboral, la impaciencia y la hostilidad. Para cada uno son elaboradas escalas específicas por la suma de la puntuación de algunos de los ítems. También se aplica una puntuación total respeto al PCTA, realizando la suma de todos los ítems, incluso los que no pertenecen a ninguna escala específica..

\section{Procedimiento}

La recogida de datos en la ciudad se realizó en la Universidad de Córdoba (UCO) y la muestra rural se recogió en diversos pueblos de la provincia de Córdoba (España). La muestra de alumnos de la UCO se recogió durante el horario lectivo de clase. El profesorado, previamente informado, y los encuestadores fueron entrenados para poder dar las instrucciones correctamente a los sujetos investigados. En el apartado de instrucciones se comentaba que era una encuesta sobre comportamientos en población joven, cuyo objetivo era la realización de una serie de actuaciones de prevención y tratamiento ante los problemas que pueden derivarse de estas conductas. Los datos serían tratados estadísticamente y se tratarían de forma completamente anónima. Cada participante firmó el consentimiento correspondiente para participar en la investigación. La recolección de los datos correspondientes a los jóvenes de la provincia de Córdoba se realizó en el tiempo máximo de un mes. Los coordinadores del 'Programa Ciudades Ante las Drogas' de cada localidad de la provincia de Córdoba pasaron las encuestas a la población joven de su pueblo o mancomunidad en clases de ciclos formativos, lugares de trabajo, gimnasios, centros sociales, etc.

\section{Análisis de datos}

El presente estudio se ha realizado mediante un diseño expost-facto prospectivo con dos grupo, en el que las variables PCTA ya han tomado su valor y se pretende investigar su relación con las conductas adictivas. El análisis de datos se dio con base a información proporcionada por los cuestionarios, buscándose evaluar las posibles relaciones entre las conductas adictivas y los patrones de personalidad Tipo A y Tipo B. Se realizó un análisis de varianza (ANOVA) con corte para el PCTA en $85 \%$ y el PCTB en $15 \%$, donde no se tuvieron en cuenta los participantes que no les caracterizaba ni PCTA ni PCTB, como ya se ha comentado en el apartado de participantes. Dicho ANOVA relacionó el PCTA y PCTB con las puntuaciones obtenidas en cada uno de los cuestionarios. Además, se realizó un análisis de Regresión Logística en el que se consideró como variable dependiente ser PCTA o PCTB y como variables independientes las diferentes puntuaciones obtenidas en los test de Internet, compras y juego.

\section{Resultados}

Teniendo en cuenta que el objetivo de este trabajo de investigación era relacionar el PCTA con conductas adictivas, como Internet, juego, compras y móvil, se ha realizado una ANOVA comparando la puntuación obtenida por cada uno de los grupos (PCTA/PCTB) en cada uno de los cuestionarios utilizados. En la figura 1 y 2, se pueden observar los resultados donde los jóvenes con personalidad PCTA obtienen una puntuación mayor en los diferentes cuestionarios. Concretamente, en el test de adicción a las 'compras', se obtiene una $\mathrm{F}=63.535, \mathrm{gl}=1$, $p<.05$; para el test de adicción a 'Internet', $\mathrm{F}=25.683$, 


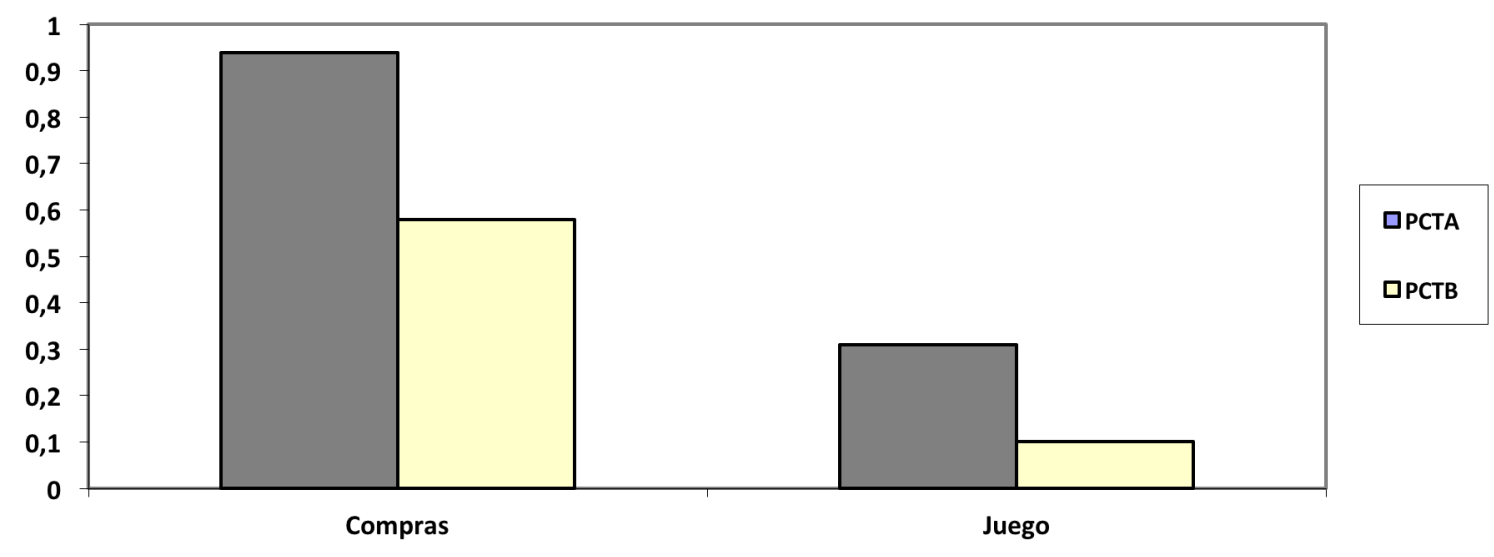

Figura 1. Comparación entre dos grupos, PCTA/PCTB con conductas adictivas: compras y juego.

$\mathrm{gl}=1, p<.05$; para el test de adicción al 'juego', $\mathrm{F}=21.289, \mathrm{gl}=1, p<.05 ; \mathrm{y}$ para el test de adicción al 'móvil, $\mathrm{F}=44.132, \mathrm{gl}=1, p<.05$. En todos los casos se dan diferencias significativas entre los grupos PCTA y PCTB.

Para determinar la fuerza de la relación entre el patrón de conducta Tipo A y la posibilidad de presentar una posible adicción conductual, se realizó un análisis de Regresión Logística Binaria. La variable dependiente fue ser PCTA o PCTB y las variables independientes las puntuaciones de los cuestionarios (adición a las compras, al juego y a Internet). Concretamente, la variable que entró a formar parte del modelo fue el test sobre adicción a las compras con una Odds Ratio superior a $1(\mathrm{OR}=1.9$, IC $95 \%=1.588-2.363$ ) indicando que están en riesgo de presentar una posible adicción a las compras 1.9 veces más los jóvenes con PCTA que los PCTB. En este caso se puede decir que las personas con PCTB tienen menos probabilidad de presentar una posible adicción a las compras.

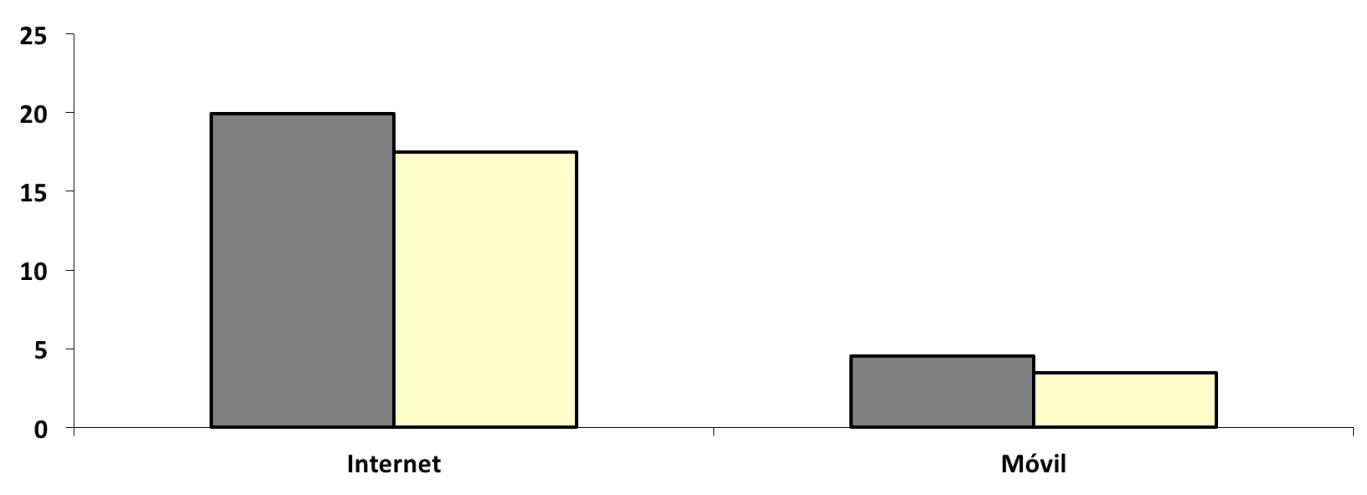

Figura 2. Comparación entre dos grupos, PCTA/PCTB con conductas adictivas: internet y móvil. 
Tabla 1

Análisis de regresión logística entre el PCTA y PCTB, y adicciones a las compras

\begin{tabular}{lcccccc}
\hline & B & E.T. & Wald & P & OR & IC 95\% \\
\hline PCTA/PCTB & & & & & & \\
Test compras & 0.661 & 0.101 & 42.513 & 0.000 & 1.937 & $1.588-2.363$ \\
\hline
\end{tabular}

\section{Discusión y Conclusiones}

Teniendo en cuenta que el objetivo de nuestra investigación era conocer la relación del PCTA y PCTB con posibles conductas adictivas como Internet, uso del móvil, compras y juego en jóvenes de la provincia de Córdoba, se puede decir que el PCTA tiene más probabilidad de presentar una posible adicción a estos comportamientos, que el PCTB. Esto coincide con otros trabajos de investigación que han relacionado el PCTA con una posible adicción a los videojuegos y al consumo de sustancias (Folson et al., 1984; Griffiths y Dancaster, 1995; Leventhal et al., 2007). Atendiendo a la regresión logística realizada, se puede decir que la puntuación del test a las compras aumenta la probabilidad de predecir la personalidad Tipo A. Es decir que una persona PCTA tiene 1.9 veces más probabilidad de presentar una posible adicción a las compras.

Los resultados de este trabajo soportan el concepto de que las actitudes de riesgo como la impulsividad y la predisposición a las adicciones entre jóvenes se correlacionan positivamente con el PCTA (Becoña y Tomás, 2010; Gutiérrez et al., 2013). Como han destacado Gómez y Fraguela (2006), los patrones de conducta son factores importantes en el discurrir del desarrollo de la vida de una persona, en todas las etapas de su vida. Los jóvenes están especialmente sujetos a presiones biológicas y sociales que les empujan al estrés, a su vez asociado directamente a conductas adictivas (Alemany, 2008)
Aunque no existe una "personalidad adictiva", hay características de personalidad como el PCTA que predisponen a una mayor vulnerabilidad individual con respecto a las conductas adictivas.

Como limitaciones determinadas por el propio diseño del estudio, destaca la recogida de datos en la propia aula de la muestra de jóvenes de la universidad, que si bien es el entorno más accesible, también conlleva un cierto sesgo de selección y una infraestimación en los resultados. Además, es necesario señalar que a pesar del interés que suscitan los resultados de la regresión logística, es necesario ser precavido a la hora de generalizar dichos resultados, ya que el tamaño de la muestra de jóvenes en riesgo es pequeño y no son clasificados correctamente. En este caso, no podemos olvidar otros factores de riesgo como los asociados al contexto social, familiar, cultural y/o hereditario de los participantes. Además, hay que profundizar más el conocimiento respecto al PCTA y el análisis de las variables aquí investigadas y sus aportaciones.

En este sentido, para futuras líneas de investigación, sería interesante replicar este tipo de trabajos con instrumentos de evaluación más adecuados que detecten aquella población susceptible de estar en riesgo; ampliar estudios que consideren los aspectos cognitivos que subyacen a estas conductas de cara a planificar actuaciones con estos jóvenes; y profundizar en las variables de protección y de riesgo que están interviniendo en la adicción de comportamientos relacionados con las compras, el juego, el uso del móvil e Internet. 


\section{Referencias}

Adan, A. (2012). Functional and dysfunctional impulsivity in young binge drinkers. Adicciones, 24(1), 17-22.

Alemany, R. N. (2008). La búsqueda de sensaciones y su relación con la vulnerabilidad a la adicción y al estrés. Adicciones, 20(1), 59-72.

American Psychiatric Association (2013). Diagnostic and Statistical Manual of Mental Disorders (5th ed.). Washington, DC: Autor.

Arteaga A., Fernández-Montalvo J. y López-Goñi J. J. (2012). Diferencias en variables de personalidad en sujetos adictos a drogas con y sin conductas violentas contra la pareja. Acción Psicológica, 9(1), 19-32. http://dx.doi.org/10.5944/ap.9.1.435

Becoña, E. (2003). Bases científicas de los programas de prevención de las drogodependencias. Madrid, España: Plan Nacional sobre Drogas.

Becoña, E. (2006). Adicción a las nuevas tecnologías. Vigo, España: Nova Galicia.

Becoña, E. y Tomás, M. C. (2010). Manual de adicciones para psicologos especializados en clinica en formación. Madrid, España: Socidrogalcohol.

Bianchi, A. y Phillips, J. G. (2005). Psychological predictors of problem mobile phone use. Cyberpsychology \& Behavior, 8, 39-51. http://dx.doi.org/10.1089/cpb.2005.8.39

Carbonell, X., Chamarro, A., Griffiths, M., Oberst, U., Cladellas, R. y Talarn, A. (2012). Problematic internet and cell phone use in spanish teenagers and young students. Anales de Psicología, 28(3), 789796.

Coll, C. y Monereo, C. (2008). Educación y aprendizaje en el siglo XX Nuevas herramientas, nuevos escenarios, nuevas finalidades. En C. Coll y C.
Monereo (Eds.), Psicología de la Educación virtual. Aprender y enseñar con las Tecnologías de la Información y la Comumcación (pp. 2-53). Madrid, España: Morata.

De la Sola Gutiérrez, S. J., Valladolid, G. R. y Fonseca, F. R. (2013). La impulsividad ¿Antesala de las adicciones comportamentales? Health and Addictions, 13(2), 145-155.

Denegri Coria, M. C., Sepúlveda Aravena, J. y Godoy Bello, M. P. (2011). Actitudes hacia la Compra y el Consumo de estudiantes de Pedagogía y profesores en ejercicio en Chile. Psicología desde el Caribe, $28,1-23$.

Diaz- Acosta, G. (2007). Evaluación de características específicas de la personalidad en diferentes trastornos físicos. Investigación en Psicología, 10(1), 173-176.

Doran, N., McChargue, D. y Cohen, L. (2007). Impulsivity and the reinforcing value of cigarette smoking. Addictive Behaviors, 32, 90-98. http://dx.doi.org/10.1016/j.addbeh.2006.03.023

Echeburúa, E. (2003). ¿Adicciones sin drogas? Las nuevas adicciones: juego, sexo, comida, compras, trabajo, Internet. Bilbao, España: Bilbao.

Echeburúa, E., y de Corral, P. (2010). Adicción a las nuevas tecnologías y a las redes sociales en jóvenes: Un nuevo reto. Adicciones, 22(2), 91-96.

Echeburúa, E., Labrador, F. J. y Becoña, E. (2009.) Adicción a las nuevas tecnologías en adolescentes y jóvenes. Madrid, España: Pirámide.

Estévez, A. (2014). Adicción al juego: últimos avances en investigación. Recuperado de http://www.ansiedadyestres.org/content/entrevistab-41

Estévez, A., Herrero, D. y Sarabia, I. (2013). Factores facilitadores de conductas adictivas de juego 
patológico en jóvenes y adolescentes [Documento técnico no publicado]. Madrid, España: ONCE.

Fantin, M. B. (2006). Perfil de personalidad y consumo de drogas en adolescentes escolarizados. Adicciones, 18(3), 285-292.

Fernández-Montalvo, J., Echeburúa Odriozola, E. y Báez Gallo, C. (1995). El cuestionario breve de juego patológico (CBJP): Un nuevo instrumento de "screening". Análisis y Modificación de Conducta, 21(76), 211-223.

Folson, A. R., Hughes, R. J., Buehler, F. J., Mittelmark, B. M., Jacobs, D. R. y Grimm, R. H. (1985). Do Type A Men Drink More Frequently than Type B Men? Findings in the Multiple Risk Factor Intervention Trial (MRFIT). Journal of Behavioral Medice, 8(3), 227-235. http://dx.doi.org/10.1007/BF00870310

García del Castillo, J. A. (2013.) Adicciones tecnológicas el auge de las redes sociales. Health and Addictions, 13(1), 5-14.

Gómez-Fraguela, J. A., Luengo-Martín, Á., RomeroTriñanes, E., Villar-Torres, P. y Sobral-Fernández, J. (2006). Estrategias de afrontamiento en el inicio de la adolescencia y su relación con el consumo de drogas y la conducta problemática. International Journal of Clinical and Health Psychology, 6(3), 581-597.

Griffiths, M. D. y Dancaster, I. (1995). The effect of type A personality on physiological arousal while playing computer games. Addictive Behaviors, 20(4), 543-548. http://dx.doi.org/10.1016/03064603(95)00001-S

Hong, F. Y., Chiu, S. I. y Huang, D. H. (2012). A model of the relationship between psychological characteristics, mobile phone addiction and use of mobile phones by Taiwanese university female students. Computers in Human Behavior, 28(6), 2152-2159.

http://dx.doi.org/10.1016/j.chb.2012.06.020
Jiménez, M. V. (2007). Personalidad, resiliencia y otros factores psicosociales asociados al consumo de sustancias psicoactivas en la adolescencia: propuesta etiológica. Revista Española de Drogodependencia, 32(3), 250-291.

Kranz, D. S., Glass, D. C. y Snyder, M. I. (1974). Helplessness, stress level, and the coronaryprone behavior pattern. Journal of Experimental Social Psychology, 10, 284-300.

Kuss, D. J., van Rooij, A. J., Shorter, G. W., Griffiths, M. D. y Van de Mheen, D. (2013). Internet addiction in adolescents: Prevalence and risk factors.Computers in Human Behavior, 29(5), 1987-1996. http://dx.doi.org/10.1016/j.chb.2013.04.002

Labrador Encinas, F. J. y Villadangos González, S. M. (2010). Adolescents and new technologies: Behaviours pointing a possible addiction problem. Psicothema, 22(2), 180-188.

Le Bon, O., Basiaux, P., Streel, E., Tecco, J., Hanaka, C., Hansenne, M., ... y Dupont, S. (2004). Personality profile and drug of choice; a multivariate analysisusing Cloninger's TCI on heroin addicts, alcoholics, and a random population group. Drug and Alcohol Dependence, 73, 175-182. http://dx.doi.org/10.1016/j.drugalcdep.2003.10.006

Leventha, M. A., Waters, A. J., Boyd, S., Moolchan, E. T., Heishman, S. J., Lerman, C. y Pickworth, W. B (2007). Associations between Cloninger's temperament dimensions and acute tobacco withdrawal. Addictive Behaviors, 32, 2976-2989. http://dx.doi.org/10.1016/j.addbeh.2007.06.014

López-Fernández, O., Honrubia-Serrano, M. y FreixaBlanxart, M. (2012). Adaptación española del "mobile phone problem use scale" para población adolescente. Adicciones, 24(2), 123-130.

Muñoz-Rivas, M. y Agustin, S. (2005). La adicción al teléfono móvil. Psicología Conductual. Revista Internacional de Psicología Clínica de la Salud, 13(3), 481-493. 
Oliva, A., Antolín-Suárez, L., Ramos, P., Jiménez, L., Jiménez-Iglesias, A., Moreno, M. C. y Hidalgo, M. V. (2013). Adicciones con y sin sustancia: paralelismo. En T. Laespada y A. Estévez, ¿Existen las adicciones sin sustancias? (pp. 87-99). Bilbao, España: Instituto Deusto de Drogodependencias. Universidad de Deusto. Recuperado de https://download.ebookshelf.de/download/0002/2881/79/L-G0002288179-0003129221.pdf

Palermo, F. y García-León, A. (1993). Metodología psicofisiológica básica en el estudio del patrón A de conducta. Revista Mexicana de Análisis de la Conducta, 19(1/2), 61-96. http://dx.doi.org/10.5514/rmac.v19.i1y2.23422

Pérez, E. J., Monje, M. T., De León, J. M. y Ruiz S. (2012). Adicción o abuso del teléfono móvil. Revisión de la literatura. Adicciones, 24(2), 139152.

Puerta-Cortés, D. X. y Carbonell, X. (2014). El modelo de los cinco grandes factores de personalidad y el uso problemático de internet en jóvenes colombianos. Adicciones, 26(1), 54-61.

Pulido, M. E. y Fonseca, J. P. (2009). Descripción y comparación de patrones de conducta, estrés laboral y bournout en personal sanitario. Hacia la Promoción de la Salud, 4(1), 109-123.

Rodríguez-Sutil, C., Gil-Corbacho, P. y Martińez Arias, R. (1996). Presentación de la escala retiro de patrón de conducta Tipo A (ERCTA). Psicothema, 8(1), 207-213.

Ruiz-Olivares, R., Lucena, V., Pino, M. J. y Herruzo, J. (2010). Análisis de comportamientos relacionados con el uso/abuso de internet, teléfono móvil, compras y juego en estudiantes universitarios. Adicciones, 22(4), 301-310.

Ruiz-Olivares, R., Lucena, V., Pino, M. J., Raya, A. y Herruzo, J. (2010). El consumo de cannabis y la percepción del riesgo en jóvenes universitarios. Psicología Conductual, 18(3), 579-590.
Salazar-Fraile, J., Ripoll-Alades, C. y Bobes, J. (2010). Narcisismo manifesto-narcisismo encubierto $y$ trastornos de personalidad en una unidad de conductas adictivas: validez predictiva de respuesta de tratamiento. Adicciones, 22,107-112.

Salehan, M. y Negahban, A. (2013). Social networking on smartphones: When mobile phones become addictive College of Business. Texas: University of North Texas.

Sánchez-Carbonell, X., Beranuy, M., Castellana, M., Chamarro, A. y Oberst, U. (2008). La adicción a Internet y al móvil: ¿Moda o trastorno? Adicciones, 20(2), 149-160.

Viñas, F. y González, M. (2010). Amenazas a través de la telefonía móvil e internet: perfil psicológico y consecuencias emocionales [Threats through mobile phones and internet: psychological profile and emotional consequences]. Acción Psicológica, 7(1), $31-40$. 